\title{
THE PROFILE NEAR BLOWUP TIME FOR SOLUTION OF THE HEAT EQUATION WITH A NONLINEAR BOUNDARY CONDITION
}

\author{
BEI HU AND HONG-MING YIN
}

\begin{abstract}
This paper studies the blowup profile near the blowup time for the heat equation $u_{t}=\Delta u$ with the nonlinear boundary condition $u_{n}=u^{p}$ on $\partial \Omega \times[0, T)$. Under certain assumptions, the exact rate of the blowup is established. It is also proved that the blowup will not occur in the interior of the domain. The asymptotic behavior near the blowup point is also studied.
\end{abstract}

\section{INTRODUCTION}

In this paper we consider the profile near the blowup time for the solution of the following problem:

$$
\begin{aligned}
\frac{\partial u}{\partial t} & =\Delta u \quad \text { for } x \in \Omega, t>0, \\
\frac{\partial u}{\partial n} & =u^{p} \quad \text { for } x \in \partial \Omega, t>0, \\
u(x, 0) & =u_{0}(x) \text { for } x \in \Omega
\end{aligned}
$$

where $\Omega$ is a bounded domain in $R^{n}$ with boundary $\partial \Omega, n$ is the exterior normal vector on $\partial \Omega, p>1$ and $u_{0}(x) \geq 0$.

It has been known for a long time (cf. [18], [19], [24]) that the problem does not have a global solution in time, for certain $u_{0}(x)$, and in [9] for all $u_{0}(x) \not \equiv 0$. Moreover, if $u^{p}$ is replaced by a general nonlinear function $f(u)$, a necessary and sufficient condition was found in [24] for the problem to have a finite time blowup. However, there are many important and interesting questions which have been open for some years. For examples, how does the solution approach the blowup time? Where is the hot spot located (blowup set)? In one space dimension as well as a radial symmetric domain in $R^{n}$, the questions were answered recently in [10], under certain monotonicity assumptions on the initial value. An improvement was given in [5] where the monotonicity condition was removed. For several space dimensions, the problem is much more challenging and there is no result except some partial answer obtained in a recent paper

Received by the editors March 18, 1993.

1991 Mathematics Subject Classification. Primary 35B35, 35B40, 35K05, 35K60.

Key words and phrases. Blowup rate, asymptotic behavior, elliptic estimates, parabolic estimates.

The first author is partially supported by U.S. National Science Foundation Grants DMS 9024986 and DMS 92-24935; the second author is partially supported by NSERC of Canada. 
[27]. To see the difficulties of the problem, let us recall some techniques used to analyze the profile of the solution near the blowup time for the following equation:

$$
u_{t}-\Delta u=u^{p} \quad(p>1) .
$$

For this equation, the above-mentioned questions were studied by a number of authors (cf. [2], [4], [11], [13]-[15], [20], [21], [25], [26], etc.). In [11] various results regarding to the blowup rate and blowup set were obtained. In deriving the blowup rate, the monotonicity of solution with respect to $t$ was imposed. Later, by introducing self-similarity variables, the authors of [14] eliminated the monotonicity condition and obtained the rate estimates if $p \in\left(1, \frac{n+2}{n-2}\right)$. Moreover, the asymptotic behavior near the blowup time was obtained in [13] and [15]. But in their proofs, the convexity of the domain is essential in order to derive the desired energy estimates. However, the convexity of the domain does not provide any help in our case since our nonlinearity is located on the boundary. By imposing the monotonicity of $u(x, t)$ with respect to $t$, one can show the energy $E(s)$ is nevertheless uniformly bounded (see (5.9) for the definition of $E(s))$. Another difficulty we encounter is the lack of results for the corresponding steady state problem. It is well known that in order to study the asymptotic behavior for the solution of an evolution equation, one needs a lot of results for the steady state equation. But to our knowledge, there are not many results available for an elliptic equation with nonlinear boundary condition in an unbounded domain. To see such an example, let us consider the following elliptic problem:

$$
\Delta w=0, \quad \text { in } \Omega, \quad \frac{\partial w}{\partial n}=w^{p}, \quad \text { on } \partial \Omega,
$$

where $\Omega=\left\{x=\left(x_{1}, x_{2}, \ldots, x_{n}\right) \in R^{n}: x_{1}>0\right\}$ and $p>1$. Obviously, there is a trivial solution $w \equiv 0$. Does the problem have a positive solution? The answer depends on the dimension $n$ and $p$. In order to show that zero is the only solution, one must study the singularity of the solution at infinity because of the unboundedness of the domain. Indeed, when $n=1$ a direct calculation concludes that zero is the only solution. When $n=2$, one can use the maximum principle to show that there is no positive solution (see Lemma 6.1 in Section 6). However, when $n>2$ and $p=\frac{n}{n-2}$ there is indeed a positive solution

$$
w(x)=\frac{c}{\left|x-x_{0}\right|^{n-2}}
$$

where $x_{0}=(-1,0, \ldots, 0)$ and $c$ is chosen properly.

By employing Kelvin's transform as in [3] and [7], we are able to show that the above elliptic problem does not have any positive solution if $p \in\left[1, \frac{n-1}{n-2}\right)$. This uniqueness result enables us to use the "localized" maximum principle, and deduce the blowup rate estimate:

$$
\max _{\Omega} u(x, t) \leq C(T-t)^{-1 /[2(p-1)]},
$$

where $T$ is the blowup time.

The second main result in this paper is concerned with blowup set. The examples in [23] indicate that the blowup may occur in the interior of the domain if the temperature on the boundary blows up at an exponential rate. 
This is not too surprising if one examines the representation of the solution and compares the boundary value with the heat kernel. Hence, in order to answer the question, we first need to show that the growth rate near the blowup time is not too fast. More precisely, we show that the rate does not exceed

$$
\frac{1}{(T-t)^{q}}
$$

for some large $q$. Then by constructing a suitable auxiliary function, we prove that the blowup only occurs on the boundary (it may occur only at a point or at every point depending on the initial data and the shape of the domain). This implies that the diffusion term is the dominating term in the interior of the domain if the heat supply is given by a power of the temperature.

The paper is written in the following manner. In Section 2, for completeness we give a new proof for the blowup property. Section 3 deals with the blowup rate of the solution. In Section 4, the blowup set is studied. The asymptotic behavior near the blowup time is considered in Section 5. The final section provides some uniqueness results for elliptic problems with a nonlinear boundary condition, which are of independent interest.

\section{BlowUP AT FINITE TIME}

Throughout this paper, we shall use $C$ and $c$ to denote various generic constants if there is no confusion. A solution of $(1.1)-(1.3)$ is always understood in the classical sense.

We assume that $\Omega$ is a bounded smooth domain in $R^{n}$. Although the following result is known (see [9], for example), we present a simple argument below. The argument will also be used in Section 4.

Theorem 2.1. For any nonzero, nonnegative initial data $u_{0}(x)$, the solution of the system (1.1) - (1.3) blows up in a finite time.

Proof. Local existence is clear. By the maximum principle, $\inf _{x \in \Omega} u(x, \varepsilon)>0$ (for small $\varepsilon>0$ ). Replacing $t=0$ by $t=\varepsilon$ if necessary, we may assume without loss of generality that $\inf _{x \in \Omega} u_{0}(x)=c>0$. Take $v(x)$ such that

$$
\begin{aligned}
& \inf _{x \in \Omega} \Delta v>0, \\
& \frac{\partial v}{\partial n}=v^{p} \quad \text { for } x \in \partial \Omega, \\
& \frac{c}{2} \leq v(x) \leq c \text { for } x \in \Omega .
\end{aligned}
$$

The existence of such a $v(x)$ can be obtained, for example, by the variational method. Then the comparison principle implies that $u(x, t) \geq \varphi(x, t)$, where $\varphi(x, t)$ is the solution of $(1.1)-(1.2)$ with initial condition $\varphi(x, 0)=v(x)$. Clearly, $\varphi_{t}(x, t) \geq 0$, by maximum principle. As a consequence, $\varphi(x, t) \geq$ $\varphi(x, 0) \geq c / 2$. Let $\psi(x, t)=\varphi_{t}(x, t)-\delta \varphi^{p}(x, t)$. Then a direct calculation shows that

$$
\begin{aligned}
& \frac{\partial \psi}{\partial t}-\Delta \psi \geq 0 \text { for } x \in \Omega, t>0 \\
& \frac{\partial \psi}{\partial n}=p u^{p} \psi \text { for } x \in \partial \Omega, t>0
\end{aligned}
$$


and $\psi(x, 0) \geq 0$ if $\delta$ is small enough. It follows that $\psi(x, t) \geq 0$, which implies that $\varphi_{t} \geq \delta \varphi^{p}$. Thus $\varphi(x, t)$ blows up in a finite time, and $u(x, t)$ must blow up at a finite time.

\section{BLOWUP RATE}

Suppose that $T$ is the blowup time. We first derive the blowup rate from above.

Theorem 3.1. Let $\Omega$ be a bounded domain in $R^{n}$ such that $\partial \Omega \in C^{2+\alpha}$ for some $0<\alpha<1$. Suppose that $1<p<\infty$ for $n=2$ and $1<p<\frac{n-1}{n-2}$ for $n \geq 3$. We assume that the initial value $u_{0} \in C^{2}(\bar{\Omega})$ satisfies

$$
\begin{aligned}
& u_{0} \geq 0, \quad \Delta u_{0} \geq 0 \quad \text { for } x \in \Omega, \\
& \frac{\partial u_{0}}{\partial n}=u_{0}^{p} \quad \text { for } x \in \partial \Omega .
\end{aligned}
$$

Then

$$
\max _{x \in \bar{\Omega}} u(x, t) \leq \frac{C}{(T-t)^{1 /[2(p-1)]}} .
$$

Remark 3.1. The restriction on $p$ comes from a nonexistence result for elliptic equations in Section 6.

Proof. Considering the equations for the functions $u$ and $u_{t}$ and using the maximum principle, we immediately obtain

$$
u(x, t) \geq 0, \quad u_{t}(x, t) \geq 0 .
$$

Thus the function $M(t)=\max _{x \in \bar{\Omega}} u(x, t)$ is monotone nondecreasing and $M(t) \rightarrow \infty$ as $t \rightarrow T-0$. The maximum principle implies that $M(t)=$ $\max _{x \in \partial \Omega} u(x, t)$.

We shall use the scaling argument analogous as in [8]. Take $T / 2<t^{*}<T$, and let $M^{*}=M\left(t^{*}\right)$. Take any point $x^{*} \in \partial \Omega$ such that $M\left(t^{*}\right)=u\left(x^{*}, t^{*}\right)$ (there may be more than one choice of such $x^{*}$ for each $t^{*}$ ) and introduce the rescaled function

$$
\varphi_{\lambda}(y, s)=\frac{1}{M^{*}} u\left(\lambda R y+x^{*}, \lambda^{2} s+t^{*}\right) \quad \text { for } y \in \overline{\Omega_{\lambda}},-\frac{T}{2 \lambda^{2}} \leq s \leq 0
$$

where $\Omega_{\lambda}=\left\{y ; \lambda R y+x^{*} \in \Omega\right\}$ and $R$ is a rotation operator such that $(-1,0,0, \ldots, 0)$ is the exterior normal vector of $\partial \Omega_{\lambda}$ at 0 . We choose $\lambda$ such that

$$
\lambda\left(M^{*}\right)^{(p-1)}=1 .
$$

Then $\varphi_{\lambda}$ solves

$$
\begin{aligned}
& \frac{\partial \varphi_{\lambda}}{\partial s}=\Delta_{y} \varphi_{\lambda} \text { for } y \in \Omega_{\lambda}, \quad-\frac{T}{2 \lambda^{2}} \leq s \leq 0, \\
& \frac{\partial \varphi_{\lambda}}{\partial n}=\varphi_{\lambda}^{p} \quad \text { for } y \in \partial \Omega_{\lambda}, \quad-\frac{T}{2 \lambda^{2}} \leq s \leq 0 \\
& \varphi_{\lambda}(0,0)=1, \\
& 0 \leq \varphi_{\lambda}(y, s) \leq 1, \quad\left(\varphi_{\lambda}\right)_{s}(y, s) \geq 0 .
\end{aligned}
$$


By assumption, the boundary $\partial \Omega_{\lambda} \cap\{|y| \leq K\}$ is uniformly in the class of $C^{2+\alpha}$ and approaches the hyperplane $\left\{y_{1}=0\right\}$ as $\lambda \rightarrow 0+$. Recalling (3.8), and using Schauder's estimates, we obtain

$$
\left\|\varphi_{\lambda}\right\|_{C^{2+\alpha, 1+(\alpha / 2)}\left(\bar{\Omega}_{\lambda} \cap\{|y| \leq K\} \times\left[-K^{2}, 0\right]\right)} \leq C_{K},
$$

where the constant $C_{K}$ is independent of $\lambda$.

Next we claim that there exist $c>0, \delta>0$ (independent of the choices of $x^{*}$ and the rotation $R$ ) such that

$$
\frac{\partial \varphi_{\lambda}}{\partial s}(0,0) \geq c \text { for } T-\delta<t^{*}<T .
$$

It is clear that $\left(\varphi_{\lambda}\right)_{s}(0,0)>0$ by Hopf's lemma. Therefore if $(3.10)$ is not true, then there exists a sequence $t_{j}^{*} \rightarrow T-0$ such that

$$
\left(\varphi_{\lambda_{j}}\right)_{s}(0,0) \rightarrow 0
$$

where $\lambda_{j}=1 /\left[M\left(t_{j}^{*}\right)\right]^{(p-1)}$. Using (3.9) and compactness, the family $\left\{\varphi_{\lambda_{j}}\right\}$ has a subsequence which converges to a function $\varphi(y, s)$. Taking this subsequence, still denoted by the subscript $\lambda_{j}$, we have

$$
\left\|\varphi_{\lambda_{j}}-\varphi\right\|_{C^{2+\beta, 1+(\beta / 2)}\left(\bar{\Omega}_{\lambda} \cap\{|y| \leq K\} \times\left[-K^{2}, 0\right]\right)} \rightarrow 0,
$$

for $0<\beta<\alpha$ and for any $K>0$. It follows that $\varphi$ satisfies

$$
\begin{aligned}
& \frac{\partial \varphi}{\partial s}=\Delta_{y} \varphi \text { for } 0<y_{1}<\infty,-\infty<s \leq 0, \\
& \frac{\partial \varphi}{\partial n}=\varphi^{p} \quad \text { for } y_{1}=0,-\infty<s \leq 0, \\
& \varphi(0,0)=1, \quad \varphi_{s}(0,0)=0 \\
& 0 \leq \varphi(y, s) \leq 1, \quad \varphi_{s}(y, s) \geq 0 .
\end{aligned}
$$

The regularity theory of parabolic PDEs implies that $\varphi$ is in $C^{\infty}$ for the $y$ and $s$ directions up to the boundary $y_{1}=0$. Differentiating (3.12) in $s$ we obtain

$$
\frac{\partial \varphi_{s}}{\partial n}=p \varphi^{p-1} \varphi_{s} \text { for } y_{1}=0,-\infty<s \leq 0,
$$

which implies that $\partial \varphi_{s}(0,0) / \partial n=0$. Notice that $\varphi_{s}(0,0)=0$ is the minimum of the function $\varphi_{s}$. It follows that $\varphi_{s}(y, s) \equiv 0$ for $s<0, y_{1}>0$ by Hopf's lemma. Thus we obtain a function $\varphi(y) \equiv \varphi(s, y)$ which satisfies the equations

$$
\begin{aligned}
& \Delta_{y} \varphi=0 \quad \text { for } 0<y_{1}<\infty, \\
& \frac{\partial \varphi}{\partial n}=\varphi^{p} \quad \text { for } y_{1}=0, \\
& \varphi(0)=1, \quad 0 \leq \varphi(y) \leq 1 .
\end{aligned}
$$

This is a contradiction to the nonexistence result which will be proved in Section

6. Thus (3.10) is established. We can rewrite $(3.10)$ as

$$
\frac{\partial u}{\partial t}\left(x^{*}, t^{*}\right) \geq c\left(M^{*}\right)^{2 p-1} \text { for } T-\delta<t^{*}<T .
$$


For each $h>0$,

$$
\frac{M\left(t^{*}+h\right)-M\left(t^{*}\right)}{h}=\frac{M\left(t^{*}+h\right)-u\left(x^{*}, t^{*}\right)}{h} \geq \frac{u\left(x^{*}, t^{*}+h\right)-u\left(x^{*}, t^{*}\right)}{h} .
$$

Letting $h \rightarrow 0+$ (noticing that $M(t)$ is Lipschitz continuous), we obtain

$$
M^{\prime}\left(t^{*}\right) \geq u_{t}\left(x^{*}, t^{*}\right) \geq c M^{2 p-1}\left(t^{*}\right) .
$$

Integrating the above equation, we conclude (3.3).

We can also get the lower bound of the blowup rate. The proof from Theorem 3.1 can be used here if we assume $u_{t} \geq 0$ (it will be true for any $1<p<\infty$ ). However, the assumption $u_{t} \geq 0$ will no longer be needed if we use the integral equation.

Theorem 3.2. Suppose that $\Omega$ is a bounded domain in $R^{n}$ such that $\partial \Omega \in C^{1+\alpha}$ for some $0<\alpha<1$, and $u_{0}(x) \geq 0$. Then

$$
\max _{x \in \bar{\Omega}} u(x, t) \geq \frac{c}{(T-t)^{1 /[2(p-1)]}} .
$$

Proof. We use the integral equation for $u(x, t)$. Let $\Gamma(x, t)$ be the fundamental solution for the heat equation, namely,

$$
\Gamma(x, t)=\frac{1}{(4 \pi t)^{(n / 2)}} \exp \left[-\frac{|x|^{2}}{4 t}\right] .
$$

Then for $0<z<t<T$ and $x \in \Omega$, we have Green's identity:

$$
\begin{aligned}
u(x, t)= & \int_{\Omega} \Gamma(x-y, t-z) u(y, z) d y \\
& +\int_{z}^{t} \int_{\partial \Omega} u^{p}(y, \tau) \Gamma(x-y, t-\tau) d S_{y} d \tau \\
& -\int_{z}^{t} \int_{\partial \Omega} u(y, \tau) \frac{\partial \Gamma}{\partial n_{y}}(x-y, t-\tau) d S_{y} d \tau .
\end{aligned}
$$

Letting $x \rightarrow \partial \Omega$ and using the jump relation for the third term on the righthand side of (3.23) (cf. [12]), we obtain

$$
\begin{array}{rlr}
\frac{1}{2} u(x, t)= & \int_{\Omega} \Gamma(x-y, t-z) u(y, z) d y \\
& +\int_{z}^{t} \int_{\partial \Omega} u^{p}(y, \tau) \Gamma(x-y, t-\tau) d S_{y} d \tau & \\
& -\int_{z}^{t} \int_{\partial \Omega} u(y, \tau) \frac{\partial \Gamma}{\partial n_{y}}(x-y, t-\tau) d S_{y} d \tau & \text { for } x \in \partial \Omega, \\
& & 0<z<t .
\end{array}
$$

We now set

$$
M(t)=\max _{x \in \bar{\Omega}} u(x, t), \quad M_{b}(t)=\max _{x \in \partial \Omega} u(x, t) .
$$


Since $\partial \Omega \in C^{1+\alpha}, \Gamma$ satisfies (cf. [12])

$$
\left|\frac{\partial \Gamma}{\partial n_{y}}(x-y, t-\tau)\right| \leq \frac{C}{(t-\tau)^{\mu}} \frac{1}{|x-y|^{n+1-2 \mu-\alpha}} \quad \text { for } x, y \in \partial \Omega .
$$

We fix $\mu$ such that $1-\alpha / 2<\mu<1$. Then (3.24) implies that

$$
\begin{aligned}
\frac{1}{2} M_{b}(t) & \leq M(z)+C \int_{z}^{t} \frac{M_{b}^{p}(\tau)}{\sqrt{t-\tau}} d \tau+C \int_{z}^{t} \frac{M_{b}(\tau)}{(t-\tau)^{\mu}} d \tau \\
& \leq M(z)+C\left(\max _{z \leq \tau \leq t} M_{b}(\tau)\right)^{p} 2 \sqrt{t-z}+C\left(\max _{z \leq \tau \leq t} M_{b}(\tau)\right)(t-z)^{1-\mu} \\
& \leq M(z)+C\left(\max _{z \leq \tau \leq t} M_{b}(\tau)\right)^{p} 2 \sqrt{T-z}+C\left(\max _{z \leq \tau \leq t} M_{b}(\tau)\right)(T-z)^{1-\mu} .
\end{aligned}
$$

For any $z \leq \eta \leq t$,

$$
\begin{aligned}
\frac{1}{2} M_{b}(\eta) & \leq M(z)+C\left(\max _{z \leq \tau \leq \eta} M_{b}(\tau)\right)^{p} 2 \sqrt{T-z}+C\left(\max _{z \leq \tau \leq \eta} M_{b}(\tau)\right)(T-z)^{1-\mu} \\
& \leq M(z)+C\left(\max _{z \leq \tau \leq t} M_{b}(\tau)\right)^{p} 2 \sqrt{T-z}+C\left(\max _{z \leq \tau \leq t} M_{b}(\tau)\right)(T-z)^{1-\mu},
\end{aligned}
$$

which implies that

$$
\begin{aligned}
\frac{1}{2} \max _{z \leq \tau \leq t} M_{b}(\tau) \leq & M(z)+C\left(\max _{z \leq \tau \leq t} M_{b}(\tau)\right)^{p} 2 \sqrt{T-z} \\
& +C\left(\max _{z \leq \tau \leq t} M_{b}(\tau)\right)(T-z)^{1-\mu} .
\end{aligned}
$$

By assumption, $T$ is the blowup time; therefore

$$
h_{z}(t) \equiv \max _{z \leq \tau \leq t} M_{b}(\tau) \rightarrow \infty \quad \text { as } t \rightarrow T-0 .
$$

Since $h_{z}(z)=M_{b}(z) \leq M(z)$, we can choose $t<T$ such that $\max _{z \leq \tau \leq t} M_{b}(\tau)$ $=4 M(z)$, and the inequality $(3.25)$ becomes

$$
M(z) \leq 2^{2 p+1} C \sqrt{T-z} M^{p}(z)+4 C(T-z)^{1-\mu} M(z) .
$$

The above inequality implies that

$$
M(z) \leq 2^{2 p+2} C \sqrt{T-z} M^{p}(z),
$$

provided $T-z$ is small enough. The theorem follows.

The integral equation (3.24) will also give us the estimate for the upper bound for the integral $\|u(\cdot, t)\|_{L^{p}(\partial \Omega)}$. There is no restriction on $p$.

Theorem 3.3. Suppose that $\Omega$ is a bounded domain in $R^{n}$ such that $\partial \Omega \in C^{1+\alpha}$ for some $0<\alpha<1, u_{0}(x) \geq 0$, and $\Delta u_{0}(x) \geq 0$. Then

$$
\left(\int_{\partial \Omega} u^{p}(x, t) d S\right)^{1 / p} \leq \frac{C}{(T-t)^{1 /[2(p-1)]}} .
$$


Proof. Let $A(t)=\|u(\cdot, t)\|_{L^{p}(\partial \Omega)}$. Under our assumptions, $u_{t} \geq 0$. It follows that $A^{\prime}(t) \geq 0$. Hölder's inequality implies that

$$
A(t) \geq c \int_{\partial \Omega} u(x, t) d S .
$$

Integrating ( 3.24 ) over $\partial \Omega$, we obtain

$$
\begin{aligned}
c \int_{\partial \Omega} u(x, t) d S & \geq c \int_{z}^{t} \frac{A^{p}(\tau)}{\sqrt{t-\tau}} d \tau-C \int_{z}^{t} \frac{A(\tau)}{|t-\tau|^{\mu}} d \tau \\
& \geq c \int_{z}^{t} \frac{A^{p}(\tau)}{\sqrt{T-\tau}} d \tau-C^{*} A(t)|T-z|^{1-\mu} .
\end{aligned}
$$

Taking $z$ so that $C^{*}|T-z|^{1-\mu}=1 / 2$, we obtain

$$
A(t) \geq c_{0} \int_{z}^{t} \frac{A^{p}(\tau)}{\sqrt{T-\tau}} d \tau \equiv c_{0} I(t) .
$$

Clearly,

$$
I^{\prime}(t)=\frac{A^{p}(t)}{\sqrt{T-t}} \geq \frac{c_{0}^{p} I^{p}(t)}{\sqrt{T-t}} .
$$

It follows that

$$
\int_{I(t)}^{I(T)} \frac{d I}{I^{p}} \geq 2 c_{0}^{p} \sqrt{T-t} .
$$

Assuming that $I(T)=\infty$ (otherwise there is nothing to prove), the above inequality implies that

$$
\begin{aligned}
I(t) & \leq 2^{-1 /(p-1)} c_{0}^{-p /(p-1)}(p-1)^{-1 /(p-1)}(T-t)^{-1 /[2(p-1)]} \\
& \equiv C^{*}(T-t)^{-1 /[2(p-1)]} .
\end{aligned}
$$

On the other hand, for $\tilde{t}=2 t-T$ (we assume that $t$ is close to $T$ here),

$$
I(t) \geq \int_{\tilde{t}}^{t} \frac{A^{p}(\tau)}{\sqrt{T-\tau}} d \tau \geq A^{p}(\widetilde{t}) \int_{2 t-T}^{t} \frac{d \tau}{\sqrt{T-\tau}}=2(\sqrt{2}-1) \sqrt{T-t} A^{p}(\widetilde{t}) .
$$

Combining this inequality with $(3.28)$, we obtain

$$
\begin{aligned}
A(\widetilde{t}) & \leq\left(C^{*}\right)^{1 / p}[2(\sqrt{2}-1)]^{-1 / p}(T-t)^{-1 /[2(p-1)]} \\
& \leq\left(C^{*}\right)^{1 / p}[2(\sqrt{2}-1)]^{-1 / p} 2^{1 /[2(p-1)]}(T-\widetilde{t})^{-1 /[2(p-1)]} .
\end{aligned}
$$

The theorem follows.

Remark 3.2. The above argument can be applied to the following integral equation:

$$
A(t)=A_{0}+\int_{0}^{t} \frac{f(A(\tau))}{\sqrt{t-\tau}} d \tau
$$

where $f(s)=s^{p}(p>1)$ and $A_{0}>0$. 
Without any condition, one can prove that $A(t)$ will blow up in a finite time. Moreover, there exist two constants $C_{1}$ and $C_{2}$ such that

$$
C_{1}(T-t)^{\frac{-1}{2(p-1)}} \leq A(t) \leq C_{2}(T-t)^{\frac{-1}{2(p-1)}},
$$

where $T$ is the blowup time.

\section{BLOWUP SET}

We shall prove in this section that the blowup will occur only at the boundary of the domain.

Theorem 4.1. Suppose that the function $u(x, t)$ is continuous on the domain $\bar{\Omega} \times[0, T)$ and satisfies

$$
\begin{aligned}
& u_{t}=\Delta u \quad \text { for }(x, t) \in \Omega \times[0, T), \\
& u \leq \frac{C}{(T-t)^{q}} \text { for }(x, t) \in \partial \Omega \times[0, T), \quad \text { for some } q>0 .
\end{aligned}
$$

Then for any $\Omega^{\prime} \subset \subset \Omega$,

$$
\sup \left\{u(x, t) ;(x, t) \in \Omega^{\prime} \times[0, T)\right\}<\infty .
$$

Proof. By approximating the domain from inside if necessary, we may assume without loss of generality that $\partial \Omega$ is smooth, say $C^{2}$.

Let $d(x)=\operatorname{dist}(x, \partial \Omega)$ and

$$
v(x)=d^{2}(x) \text { for } x \in N_{\varepsilon}(\partial \Omega),
$$

where $N_{\varepsilon}(\partial \Omega)=\left\{(x \in \Omega, d(x)<\varepsilon\}\right.$. Since $\partial \Omega$ is $C^{2}$, the function $v(x)$ is in $C^{2}\left(\overline{N_{\varepsilon}(\partial \Omega)}\right)$ if $\varepsilon$ is small enough. Clearly,

$$
\Delta v-\frac{(q+1)|\nabla v|^{2}}{v}=2-4(q+1) \quad \text { on } \partial \Omega .
$$

Since $v \in C^{2}\left(\overline{N_{\varepsilon}(\partial \Omega)}\right)$,

$$
\Delta v-\frac{(q+1)|\nabla v|^{2}}{v} \geq-4(q+1) \quad \text { in } \overline{N_{\varepsilon_{0}}(\partial \Omega)}
$$

if $\varepsilon_{0}$ is small enough. We next extend $v(x)$ to a function on $\bar{\Omega}$ such that $v \in C^{2}(\bar{\Omega})$ and $v \geq c_{0}>0$ on $\overline{\Omega \backslash N_{\varepsilon_{0}}(\partial \Omega)}$. Then

$$
\Delta v-\frac{(q+1)|\nabla v|^{2}}{v} \geq-C^{*} \quad \text { on } \bar{\Omega}
$$

for some $C^{*}>0$. Set

$$
w(x, t)=\frac{C_{1}}{\left[v(x)+C^{*}(T-t)\right]^{q}} .
$$

Then

$$
w_{t}-\Delta w=\frac{C_{1} q}{\left[v(x)+C^{*}(T-t)\right]^{(q+1)}}\left(C^{*}+\Delta v-\frac{(q+1)|\nabla v|^{2}}{v+C^{*}(T-t)}\right)>0 .
$$


Take $C_{1}$ to be large enough so that $w(x, 0) \geq u(x, 0)$ and $C_{1} \geq\left(C^{*}\right)^{q}$. Then the maximum principle implies that $w(x, t) \geq u(x, t)$, and

$$
\sup \left\{u(x, t) ;(x, t) \in \Omega^{\prime} \times[0, T)\right\} \leq C_{1} \sup \left\{\frac{1}{v^{q}(x)} ; x \in \Omega^{\prime}\right\}<\infty .
$$

Corollary 4.2. Assume that $u_{0}(x) \geq 0$ and $\Delta u_{0}(x) \geq 0$ (which implies that $\left.u_{t} \geq 0\right)$. Then no blowup will occur in the interior of the domain $\Omega$.

Proof. As in the proof of Theorem 2.1, we can easily establish $u_{t} \geq \delta u^{p}$ for $t \geq \varepsilon$ for small $\delta$ and $\varepsilon$. It then follows that

$$
\max _{x \in \bar{\Omega}} u(x, t) \leq \frac{C}{(T-t)^{1 /(p-1)}} .
$$

Although ( 4.1 ) does not give us the best rate at the boundary, it is enough for us to apply Theorem 4.1 .

\section{ASYMPTOTIC BEHAVIOR NEAR THE BLOWUP POINT}

We first establish some preliminary estimates. We will assume throughout this section that

$$
\max _{x \in \bar{\Omega}} u(x, t) \leq \frac{C}{(T-t)^{\beta}}, \quad \beta=\frac{1}{2(p-1)} .
$$

This condition will be satisfied if the assumptions of Theorem 3.1 are in force. As in Giga and Kohn [14], we introduce the scaled solution:

$$
\begin{aligned}
& w(y, s)=(T-t)^{\beta} u(x, t), \\
& x-a=y \sqrt{T-t}, \quad T-t=e^{-s},
\end{aligned}
$$

where $a$ is a fixed point on $\partial \Omega$. If $u$ solves (1.1), then $w$ solves

$$
\frac{\partial}{\partial s} w-\Delta w+\frac{1}{2} y \cdot \nabla w+\beta w=0
$$

in the domain

$$
W=\bigcup_{s>s_{0}} \Omega(s)
$$

where

$$
\Omega(s)=\left\{(y, s) ; e^{-s / 2} y+a \in \Omega\right\}, \quad s_{0}=-\ln T .
$$

From the proof of Theorem 3.1 (cf. (3.9)) we have the following estimates:

$$
\begin{aligned}
& \left|\frac{\partial}{\partial x_{j}} u(x, t)\right| \leq C \max _{x \in \bar{\Omega}} u^{p}(x, t) \leq \frac{C}{(T-t)^{p \beta}}, \\
& \left|\frac{\partial^{2}}{\partial x_{j} \partial x_{k}} u(x, t)\right| \leq C \max _{x \in \bar{\Omega}} u^{2 p-1}(x, t) \leq \frac{C}{(T-t)^{(2 p-1) \beta}} .
\end{aligned}
$$


As a consequence,

$$
\begin{aligned}
& 0 \leq w(y, s) \leq C \quad \text { for }(y, s) \in W \\
& \left|\frac{\partial}{\partial y_{j}} w(y, s)\right| \leq C \quad \text { for }(y, s) \in W \\
& \left|\frac{\partial^{2}}{\partial y_{j} \partial y_{k}} w(y, s)\right| \leq C \quad \text { for }(y, s) \in W .
\end{aligned}
$$

We need the following formulae:

Lemma 5.1. If $f(s, y): W \mapsto R$ is a smooth function, then

$$
\begin{aligned}
\frac{d}{d s} \int_{\Omega(s)} f(s, y) d y= & \int_{\Omega(s)} f_{s}(s, y) d y+\frac{1}{2} \int_{\partial \Omega(s)} f(s, y)(y \cdot \mathbf{n}) d S \\
\frac{d}{d s} \int_{\partial \Omega(s)} f(s, y) d S= & \int_{\partial \Omega(s)} f_{s}(s, y) d S \\
& +\frac{1}{2} \int_{\partial \Omega(s)}[(n-1) f(s, y)+\nabla f(s, y) \cdot y] d S
\end{aligned}
$$

where $\mathbf{n}$ is the exterior normal vector of $\partial \Omega(s)$ and $d S$ is the surface area element.

Proof. The proof of (5.6) can be found in Liu [20], and ( 5.7 ) can be proved in a similar manner.

Let us rewrite equation (5.2) in divergence form:

$$
\rho w_{s}-\nabla \cdot(\rho \nabla w)+\beta \rho w=0,
$$

where $\rho=e^{-|y|^{2} / 4}$. Now we introduce the "energy" functional as in Giga and Kohn [14]:

$$
E(s)=\frac{1}{2} \int_{\Omega(s)}\left(\rho|\nabla w|^{2}+\beta \rho w^{2}\right) d y-\frac{1}{p+1} \int_{\partial \Omega(s)} \rho w^{p+1} d S .
$$

First of all, multiplying ( 5.8 ) with $w_{s}$ and integrating over $\Omega(s)$, we have the following identity:

$$
\int_{\Omega(s)} \rho\left|w_{s}\right|^{2} d y+\int_{\Omega(s)}\left[\rho \nabla w \nabla w_{s}+\beta \rho w w_{s}\right] d y=\int_{\partial \Omega(s)} \rho w^{p} w_{s} d s .
$$

Using formulae $(5.6)-(5.7)$ and the above identity, we now obtain

$$
\begin{aligned}
E^{\prime}(s)= & \int_{\Omega(s)}\left(\rho \nabla w \nabla w_{s}+\beta \rho w w_{s}\right) d y+\frac{1}{4} \int_{\partial \Omega(s)}\left(\rho|\nabla w|^{2}+\beta \rho w^{2}\right)(y \cdot \mathbf{n}) d S \\
& -\int_{\partial \Omega(s)} \rho w^{p} w_{s} d S-\frac{1}{2(p+1)} \int_{\partial \Omega(s)}\left[(n-1) \rho w^{p+1}+\nabla\left(\rho w^{p+1}\right) \cdot y\right] d S \\
= & -\int_{\Omega(s)} \rho\left|w_{s}\right|^{2} d y+J(s)
\end{aligned}
$$


where

$$
\begin{aligned}
J(s)= & \frac{1}{4} \int_{\partial \Omega(s)}\left(\rho|\nabla w|^{2}+\beta \rho w^{2}\right)(y \cdot \mathbf{n}) d S \\
& -\frac{1}{2(p+1)} \int_{\partial \Omega(s)}\left[(n-1) \rho w^{p+1}+\nabla\left(\rho w^{p+1}\right) \cdot y\right] d S .
\end{aligned}
$$

The estimate on $J(s)$ is crucial for taking the limit as $s \rightarrow \infty$.

Lemma 5.2. There exists a constant $C^{*}$ such that

$$
J(s)<C^{*} e^{-s / 4} .
$$

Proof. Without loss of generality, we may assume that $a=0 \in \partial \Omega$ and $(-1,0, \ldots, 0)$ is the exterior unit normal vector of $\Omega$ at 0 . Then $\partial \Omega$ near 0 is represented by the formula

$$
x_{1}=g\left(x_{2}, x_{3}, \ldots, x_{n}\right) \text { for } x_{1}^{2}+\cdots+x_{n}^{2} \leq \delta^{2},
$$

where $g \in C^{2+\alpha}$ and $g(0)=0, \nabla g(0)=0$. We can represent $\partial \Omega(s)$ by

$$
y_{1}=e^{s / 2} g\left(y^{\prime} e^{-s / 2}\right) \equiv G\left(y^{\prime}\right) \quad \text { for }\left|y^{\prime}\right| \leq \delta e^{s / 2}, \quad y^{\prime}=\left(y_{2}, y_{3}, \ldots, y_{n}\right) .
$$

Clearly,

$$
\begin{gathered}
G(0)=0, \quad \nabla G(0)=0, \quad\left|G_{y_{j} y_{k}}\right| \leq C e^{-s / 2} \quad(2 \leq k, j \leq n), \\
\mathbf{n}=\left(\frac{-1}{\sqrt{1+|\nabla G|^{2}}}, \frac{\nabla G}{\sqrt{1+|\nabla G|^{2}}}\right), \quad d S=\sqrt{1+|\nabla G|^{2}} d y^{\prime}, \\
y \cdot \mathbf{n}=\frac{-G+y_{2} G_{y_{2}}+\cdots+y_{n} G_{y_{n}}}{\sqrt{1+|\nabla G|^{2}}}
\end{gathered}
$$

From now on, the generic constants $C$ will depend only on the given data like $\partial \Omega, u_{0}(x)$, and $p$.

We split the integrals in $J(s)$ on $\partial \Omega(s)$ into $\partial \Omega(s) \cap\left\{\left|y^{\prime}\right| \leq \delta e^{s / 8}\right\}$ and $\partial \Omega(s) \cap\left\{\left|y^{\prime}\right|>\delta e^{s / 8}\right\}$. Recalling (5.3)-(5.5),

$\mid$ the integrals in $J(s)$ on $\partial \Omega(s) \cap\left\{\left|y^{\prime}\right|>\delta e^{s / 8}\right\} \mid$

$$
\begin{aligned}
& \leq C \int_{\partial \Omega(s) \cap\left\{\left|y^{\prime}\right|>\delta e^{s / 8}\right\}} e^{-|y|^{2} / 8} d S \\
& \leq C \exp \left(-\frac{1}{8} \delta^{2} e^{s / 8}\right) \cdot|\partial \Omega(s)| \\
& \leq C \exp \left(-\frac{1}{8} \delta^{2} e^{s / 8}+\frac{(n-1) s}{2}\right) .
\end{aligned}
$$

Now we write

the integrals in $J(s)$ on $\partial \Omega(s) \cap\left\{\left|y^{\prime}\right| \leq \delta e^{s / 8}\right\} \equiv I_{1}(s)+I_{2}(s)$, 
where $I_{1}(s)$ is estimated as follows:

$$
\begin{aligned}
\left|I_{1}(s)\right| & =\frac{1}{4}\left|\int_{\partial \Omega(s) \cap\left\{\left|y^{\prime}\right| \leq \delta e e^{s / 8}\right\}}\left(\rho|\nabla w|^{2}+\beta \rho w^{2}\right)(y \cdot \mathbf{n}) d S\right| \\
& \leq C \int_{\partial \Omega(s) \cap\left\{\left|y^{\prime}\right| \leq \delta e^{s / 8}\right\}} \rho|(y \cdot \mathbf{n})| d S \\
& \leq C \int_{\left\{\left|y^{\prime}\right| \leq \delta e^{s / 8}\right\}} \rho\left(\left|G-\nabla G \cdot y^{\prime}\right|\right) d y^{\prime} \\
& \leq C \int_{\left\{\left|y^{\prime}\right| \leq \delta e^{s / 8}\right\}} \rho\left|y^{\prime}\right|^{2} \max \left|G_{y_{j} y_{k}}\right| d y^{\prime} \\
& \leq C e^{-s / 2}\left(e^{s / 8}\right)^{2} \int_{\left\{\left|y^{\prime}\right| \leq \delta e^{s / 8}\right\}} \rho d y^{\prime} \\
& \leq C e^{-s / 4}
\end{aligned}
$$

To estimate $I_{2}(s)$, we let $\varphi(y, s)=\rho w^{p+1}$ and compute

$$
\begin{aligned}
2(p-1)\left|I_{2}(s)\right| & \\
= & \left|\int_{\partial \Omega(s) \cap\left\{\left|y^{\prime}\right| \leq \delta e^{s / 8}\right\}}\left[(n-1) \rho w^{p+1}+\nabla\left(\rho w^{p+1}\right) \cdot y\right] d S\right| \\
& =\left|\int_{\left\{\left|y^{\prime}\right| \leq \delta e^{s / 8}\right\}}\left[(n-1) \varphi+\left(\varphi_{y_{1}} G+\varphi_{y_{2}} y_{2}+\cdots+\varphi_{y_{n}} y_{n}\right)\right] \sqrt{1+|\nabla G|^{2}} d y^{\prime}\right| \\
& =\mid \int_{\left\{\left|y^{\prime}\right| \leq \delta e^{s / 8}\right\}}\left[(n-1) \varphi+\sum_{k=2}^{n}\left(\varphi_{y_{1}} G_{y_{k}}+\varphi_{y_{k}}\right) y_{k}\right. \\
& \left.+\varphi_{y_{1}}\left(G-\sum_{k=2}^{n} y_{k} G_{y_{k}}\right)\right] \sqrt{1+|\nabla G|^{2}} d y^{\prime} \mid \\
& \leq K_{1}(s)+K_{2}(s),
\end{aligned}
$$

where

$$
\begin{aligned}
K_{2}(s) & =\int_{\left\{\left|y^{\prime}\right| \leq \delta e^{s / 8}\right\} \mid}\left|\varphi_{y_{1}}\left(G-\sum_{k=2}^{n} y_{k} G_{y_{k}}\right)\right| \sqrt{1+|\nabla G|^{2}} d y^{\prime} \\
& \leq C \int_{\left\{\left|y^{\prime}\right| \leq \delta e^{s / 8}\right\}} e^{-\left|y^{\prime}\right|^{2} / 8}\left|y^{\prime}\right|^{2} \max \left|G_{y_{j} y_{k}}\right| d y^{\prime} \\
& \leq C e^{-s / 2}\left(e^{s / 8}\right)^{2} \int_{R^{n-1}} e^{-\left|y^{\prime}\right|^{2} / 8} d y^{\prime} \\
& \leq C e^{-s / 4}
\end{aligned}
$$


Finally, let us estimate $K_{1}(s)$ using integration by parts on the $(n-1)$ dimensional disk:

$$
\begin{aligned}
K_{1}(s)= & \left|\int_{\left\{\left|y^{\prime}\right| \leq \delta e^{s / 8}\right\}}\left[(n-1) \varphi+\sum_{k=2}^{n}\left(\varphi_{y_{1}} G_{y_{k}}+\varphi_{y_{k}}\right) y_{k}\right] \sqrt{1+|\nabla G|^{2}} d y^{\prime}\right| \\
= & \mid \int_{\left\{\left|y^{\prime}\right| \leq \delta e^{s / 8}\right\}}\{(n-1) \varphi \\
& \left.\quad+\sum_{k=2}^{n} y_{k} \frac{\partial}{\partial y_{k}}\left(\varphi\left[G\left(y_{2}, \ldots, y_{n}\right), y_{2}, \ldots, y_{n}\right]\right)\right\} \sqrt{1+|\nabla G|^{2}} d y^{\prime} \mid \\
= & \mid \int_{\left\{\left|y^{\prime}\right|=\delta e^{s / 8}\right\}} \varphi \sum_{k=2}^{n} y_{k} \frac{y_{k}}{\left|y^{\prime}\right|} \sqrt{1+|\nabla G|^{2}} d y^{\prime} \\
& \quad-\int_{\left\{\left|y^{\prime}\right| \leq \delta e^{s / 8}\right\}} \varphi \sum_{k=2}^{n} y_{k} \frac{\partial}{\partial y_{k}}\left(\sqrt{1+|\nabla G|^{2}}\right) d y^{\prime} \mid \\
\leq & C e^{s / 8} \int_{\left\{\left|y^{\prime}\right|=\delta e^{s / 8}\right\}} \rho d y^{\prime}+C e^{-s / 2} \int_{\left\{\left|y^{\prime}\right| \leq \delta e^{s / 8}\right\}} \rho\left|y^{\prime}\right| d y^{\prime} \\
\leq & C e^{s / 8} \exp \left(-\frac{1}{4} \delta^{2} e^{s / 4}+\frac{(n-2) s}{2}\right)+C e^{-s / 2} .
\end{aligned}
$$

Combining the estimates from ( 5.14$)-(5.18)$, we conclude the lemma.

Remark 5.1. The estimates are uniformly valid for $a \in \partial \Omega$.

From Lemma 5.2 and (5.10),

$$
\frac{d}{d s}\left(E(s)+4 C^{*} e^{-s / 4}\right) \leq 0 .
$$

It follows that the function $E(s)+4 C^{*} e^{-s / 4}$ is monotone decreasing. Clearly

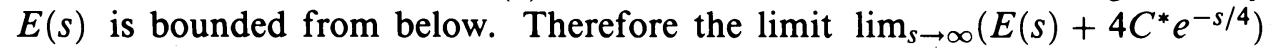
exists. So the limit

$$
\lim _{s \rightarrow \infty} E(s)=E(\infty) \text { exists. }
$$

Integrating (5.10), we also conclude

\section{Lemma 5.3.}

$$
\int_{s_{0}}^{\infty} \int_{\Omega(s)} \rho\left|w_{s}\right|^{2} d y d s \leq C<\infty
$$

Notice that ( 5.19 ) claims that the "energy $E(s)$ " has a limit. The energy $E(s)$ is the difference of two terms. In fact, the limits of both terms exist. Let

$$
\begin{aligned}
& E_{1}[w](s)=\int_{\Omega(s)}\left(\rho|\nabla w|^{2}+\beta \rho w^{2}\right) d y, \\
& E_{2}[w](s)=\int_{\partial \Omega(s)} \rho w^{p+1} d S .
\end{aligned}
$$




\section{Theorem 5.4.}

$$
\lim _{s \rightarrow \infty} E_{1}[w](s)=\frac{2(p+1)}{p-1} E(\infty), \quad \lim _{s \rightarrow \infty} E_{2}[w](s)=\frac{2(p+1)}{p-1} E(\infty) .
$$

Proof. By ( 5.19)

$$
\lim _{s \rightarrow \infty}\left(\frac{1}{2} E_{1}(s)-\frac{1}{p+1} E_{2}(s)\right)=E(\infty)
$$

By $(5.3)-(5.5)$, we can take a subsequence $s_{j} \rightarrow \infty$ such that $w\left(y, s+s_{j}\right)$ converges weakly to a function $w_{\infty}(y, s)$. Lemma 5.3 implies that $w_{\infty}$ is independent of $s$. Hence

$$
\begin{aligned}
& -\nabla\left(\rho \nabla w_{\infty}\right)+\beta \rho w_{\infty}=0 \text { for } y_{1}>0,-\infty<y_{k}<\infty(2 \leq k \leq n), \\
& \frac{\partial w_{\infty}}{\partial n}=w_{\infty}^{p} \quad \text { on } y_{1}=0 .
\end{aligned}
$$

It follows that $E_{1}[w]\left(s+s_{j}\right) \rightarrow c_{1}$ and $E_{2}[w]\left(s+s_{j}\right) \rightarrow c_{2}$ as $s_{j} \rightarrow \infty$, where $c_{1}=E_{1}\left[w_{\infty}\right]$ and $c_{2}=E_{2}\left[w_{\infty}\right]$. Multiplying (5.22) by $w_{\infty}$ and integrating, we obtain

$$
c_{2}-c_{1}=0 \text {. }
$$

Combining it with $(5.21)$ we find that

$$
c_{1}=c_{2}=\frac{2(p+1)}{p-1} E(\infty) \text {. }
$$

Since $c_{1}$ and $c_{2}$ are uniquely determined by $E(\infty)$, the limits

$$
\lim _{s_{j} \rightarrow \infty} E_{1}[w]\left(s+s_{j}\right) \quad \text { and } \lim _{s_{j} \rightarrow \infty} E_{2}[w]\left(s+s_{j}\right)
$$

are actually independent of the choices of the subsequence $s_{j}$. The theorem is proved.

As in the proof of Giga and Kohn [14], $w(y, s)$ will have to converge to $w_{\infty}$ uniformly on any bounded subset as $s \rightarrow \infty$, if the solution $w_{\infty}$ in (5.22)(5.23) is independent of the choices of the subsequence $s_{j}$. This is the case if the positive solution to the system is unique.

Theorem 5.5. Let the assumption ( 5.1 ) be in force. If a bounded positive solution with bounded gradient to the system

$$
\begin{aligned}
& -\Delta \psi+\frac{1}{2} y \cdot \nabla \psi+\beta \psi=0 \text { for } y_{1}>0,-\infty<y_{k}<\infty(2 \leq k \leq n) \\
& -\frac{\partial \psi}{\partial y_{1}}=\psi^{p} \text { for } y_{1}=0,-\infty<y_{k}<\infty(2 \leq k \leq n)
\end{aligned}
$$

is unique, then for the solution $u$ of ( 1.1 ) and $a \in \partial \Omega$ (assuming without loss of generality that the exterior normal at the point $a$ is $(-1,0, \ldots, 0))$,

$$
\lim _{t \rightarrow T-0}(T-t)^{\beta} u(a+y \sqrt{T-t}, t)
$$

exists and equals either 0 or $\psi$. The limit in (5.26) is taken uniformly on each bounded set $|y| \leq C$. 
The one-dimensional positive solution (i.e., the solution which is a function of $y_{1}$ only) of $(5.24)-(5.25)$ is unique [10]. Therefore we have the following corollary, which is a slight generalization (the assumptions on the sign of third and fourth order derivatives of the initial data are no longer required here) of the main result proved in [10]. The corollary does not assume (5.1).

Corollary 5.6. Suppose that $\Omega=B_{R}(0)$ is a ball and that the initial data is radially symmetric with $u_{0} \geq 0, u_{0} \not \equiv 0$ and $\Delta u_{0} \geq 0$. Then for the solution $u$ of $(1.1)$ and $a=(-R, 0, \ldots, 0) \in \partial \Omega$,

$$
\lim _{t \rightarrow T-0}(T-t)^{\beta} u(a+y \sqrt{T-t}, t)
$$

exists and equals $\psi$, where $\psi$ is the one-dimensional positive solution. The limit in ( 5.27 ) is taken uniformly on each bounded set $|y| \leq C$.

Proof. We need assumption (5.1) in order to use Theorem 5.5. Clearly, (5.1) is valid if Theorem 3.1 is valid. We first claim that Theorem 3.1 is valid in this radially symmetric case without the restriction on $p$. The restriction on $p$ comes from the nonexistence result for the system (3.16)-(3.18). In the radially symmetric case, it is easy to see that the function $\varphi$ in $(3.16)-(3.18)$ is a function of the $y_{1}$ variable only, and hence we obtain a contradiction immediately. This proves the claim.

Now it suffices to prove that the solution $w_{\infty}$ is independent of the choices of the subsequence $s_{j}$. Since $w_{\infty}$ is independent of the variables $\left(y_{2}, \ldots, y_{n}\right)$ on $y_{1}=0$, the maximum principle implies that $\left(w_{\infty}\right)_{y_{k}} \equiv 0$ for $k=2, \ldots, n$. Therefore $w_{\infty}$ is uniquely determined as a one-dimensional solution of $y_{1}$.

Since the solution is now radially symmetric, the maximum of the solution $u(r, t)$ is attained at the boundary $\partial \Omega$. Therefore Theorem 3.2 yields that the limit in (5.27) is nonzero.

Remark 5.2. If the positive solutions of ( 5.24$)$ - ( 5.25$)$ are "discrete" (which will guarantee that $w_{\infty}$ is independent of the choices of $\left.s_{j}\right)$, then the limit in ( 5.26 ) will converge to one of the nonnegative solutions. By "discrete" we mean that $\left\|\psi_{1}-\psi_{2}\right\| \geq c_{0}>0$ for any two distinct solutions $\psi_{1}$ and $\psi_{2}$, where $c_{0}$ is independent of the solutions. The norm $\|\cdot\|$ could be any norms like $\|\psi\|=\|\rho \psi\|_{L^{2}},\|\psi\|=\|\rho \psi\|_{L^{2}}+\|\rho \nabla \psi\|_{L^{2}}$, or $\|\psi\|=\|\psi\|_{L^{\infty}}$.

\section{NONEXISTENCE RESULT FOR THE ELLIPTIC EQUATIONS}

In this section we prove the nonexistence result used in Section 3. We first consider the case $n=2$; the proof is very simple.

Lemma 6.1. Suppose that $n=2$ and

$$
\begin{aligned}
& \Delta_{y} \varphi=0 \text { for } 0<y_{1}<\infty,-\infty<y_{2}<\infty, \\
& -\frac{\partial \varphi}{\partial y_{1}} \geq 0 \text { for } y_{1}=0, \\
& 0 \leq \varphi(y) \leq 1 .
\end{aligned}
$$

Then

$$
\varphi(y) \equiv \text { constant } .
$$


Proof. Take $y_{0} \in[0, \infty) \times(-\infty, \infty)$. For any $0<\varepsilon<1,0<\delta<1$, we construct the auxiliary function

$$
\psi(y)=\varepsilon \log \left(\frac{\left|y-y_{0}\right|^{2}}{\delta^{2}}\right)+C_{\delta},
$$

where $C_{\delta}=\max _{\left|y-y_{0}\right|=\delta}\left[\varphi\left(y_{0}\right)-\varphi(y)\right]$. Note that $\psi=C_{\delta}$ on $\left\{\left|y-y_{0}\right|=\delta\right\}$, $\left.\psi(y)>1 \geq \varphi_{(} y_{0}\right)-\varphi(y)$ on $\left|y-y_{0}\right|=e^{1 / \varepsilon}$. A direct calculation also shows that $-\psi_{y_{1}}\left(0, y_{2}\right) \geq 0$. Thus by the maximum principle,

$$
\varphi\left(y_{0}\right)-\varphi(y) \leq \psi(y) \text { in the region }\left\{y_{1}>0, \delta<\left|y-y_{0}\right|<e^{1 / \varepsilon}\right\} .
$$

Letting $\varepsilon \rightarrow 0+$ and then $\delta \rightarrow 0+$, we conclude $\varphi\left(y_{0}\right)-\varphi(y) \leq 0$ for any $y$ and $y_{0}$.

Lemma 6.2. Suppose that $n \geq 3, p<\frac{n-1}{n-2}$, and

$$
\begin{aligned}
& \Delta_{y} \varphi=0 \text { for } 0<y_{1}<\infty,-\infty<y_{k}<\infty(2 \leq k \leq n), \\
& -\frac{\partial \varphi}{\partial y_{1}}=\varphi^{p} \quad \text { for } y_{1}=0, \\
& \varphi(y) \geq 0 .
\end{aligned}
$$

Then

$$
\varphi(y) \equiv 0 .
$$

Proof. Assume on the contrary that $\varphi \not \equiv 0$. Then we claim that

$$
\varphi(y) \geq \frac{c}{|y|^{n-2}} \text { for } y_{1}>0,1 \leq|y|<\infty,
$$

for some $c>0$. In fact, if we take $c=\min _{|y|=1} \varphi(y)$, then by the maximum principle, $c>0$. The function $w(|y|)=c /|y|^{n-2}$ satisfies

$$
\begin{aligned}
& \Delta w=0 \text { for } 0<y_{1}<\infty, 1 \leq|y|<\infty, \\
& -\frac{\partial w}{\partial y_{1}}=0 \leq-\frac{\partial \varphi}{\partial y_{1}} \text { for } y_{1}=0, \\
& w(y) \leq \varphi(y) \quad \text { on }|y|=1 .
\end{aligned}
$$

Therefore by the maximum principle,

$$
w(|y|)-w(R) \leq \varphi(y) \text { for } 0<y_{1}<\infty, 1 \leq|y|<R .
$$

Letting $R \rightarrow \infty$, we obtain (6.9).

In order to study the behavior of $\varphi(y)$ near $\infty$, we introduce the Kelvin inversion:

$$
\psi(z)=|y|^{n-2} \varphi(y), \quad z=\frac{y}{|y|^{2}} .
$$

The function $\psi(z)$ may have a singularity at 0 . It satisfies the equations:

$$
\Delta \psi=0 \text { for } 0<z_{1}<\infty,-\infty<z_{k}<\infty(2 \leq k \leq n),
$$

(6.11) $-\frac{\partial \psi}{\partial z_{1}}=|z|^{-\alpha} \psi^{p} \quad$ for $z_{1}=0,|z|>0, \quad$ where $\alpha=n-p(n-2)$. 
Recalling (6.9), we have

$$
\psi(z) \geq c>0 \text { for } 0 \leq z_{1} \leq 1,0<|z| \leq 1 .
$$

For any $\varepsilon>0$, we take a smooth cut-off function $\zeta(z)$ such that

$$
\begin{aligned}
& \zeta(z)=0 \text { for }|z| \leq \varepsilon \text { and }|z| \geq 4 \varepsilon, \\
& \zeta(z)=1 \text { for } 2 \varepsilon \leq|z| \leq 3 \varepsilon, \\
& 0 \leq \zeta(z) \leq 1, \quad|\nabla \zeta(z)| \leq \frac{C}{\varepsilon} .
\end{aligned}
$$

Multiplying equation (6.10) with $\zeta^{2} \psi^{-1}$ and integrating over $\Omega=\left\{z_{1}>\right.$ $0,0<|z|<1\}$, we obtain

$$
\begin{aligned}
\int_{\Omega} \zeta^{2} & \frac{|\nabla \psi|^{2}}{|\psi|^{2}} d z+\int_{\left\{z_{1}=0\right\}} \zeta^{2}|z|^{-\alpha} \psi^{p-1} d S \\
& =2 \int_{\Omega} \frac{\zeta}{\psi} \nabla \zeta \nabla \psi d z \leq \frac{1}{2} \int_{\Omega} \zeta^{2} \frac{|\nabla \psi|^{2}}{|\psi|^{2}} d z+2 \int_{\Omega}|\nabla \zeta|^{2} d z .
\end{aligned}
$$

Hence

$$
\int_{\left\{z_{1}=0\right\}} \zeta^{2}|z|^{-\alpha} \psi^{p-1} d S \leq 2 \int_{\Omega}|\nabla \zeta|^{2} d z
$$

which implies that

$$
\varepsilon^{n-1} \varepsilon^{-\alpha} \leq C \varepsilon^{n} \frac{1}{\varepsilon^{2}}
$$

Noticing that $\alpha>1$, we obtain a contradiction if $\varepsilon$ is small enough.

Remark 6.1. The restriction on $p$ in Lemma 6.2 may not be optimal. We conjecture that the uniqueness holds for $1<p<\frac{n}{n-2}$ if $n>2$.

Note. The uniqueness for $1<p<\frac{n}{n-2} \quad(n>2)$ is indeed valid. The detail of the proof is given by the first author in a paper which appeared in Differential and Integral Equations 7 (1994), pp. 301-313.

\section{ACKNOWLEDGMENT}

The authors would like to thank the referee for helpful suggestions. The referee suggested to us that the limit in (5.27) is nonzero and should be included in Corollary 5.6.

\section{REFERENCES}

1. H. Amann, Parabolic evolution equations and nonlinear boundary conditions, J. Differential Equations 72 (1988), 201-269.

2. J. Bebernes and D. Eberly, Mathematical problems from combustion theory, Springer-Verlag, New York, 1989.

3. L. A. Caffarelli, B. Gidas, and J. Spruck, Asymptotic symmetry and local behavior of semilinear elliptic equations with critical Sobolev growth, Comm. Pure Appl. Math. 42 (1989), 271-297.

4. L. A. Caffarelli and A. Friedman, Blowup of solutions of nonlinear heat equations, J. Math. Anal. Appl. 129 (1988), 409-419.

5. J. M. Chadam and H. M. Yin, A diffusion equation with localized chemical reactions, Proc. Edinburgh Math. Soc. 37 (1993), 101-118. 
6. M. Chipot, M. Fila, and P. Quittner, Stationary solutions, blowup and convergence to stationary solutions for semilinear parabolic equations with nonlinear boundary conditions, Acta Math. Univ. Comenian. 60 (1991), 35-103.

7. B. Gidas and J. Spruck, Global and local behavior of positive solutions of nonlinear elliptic equations, Comm. Pure Appl. Math. 34 (1981), 525-598.

8. $ـ$ A priori bounds for positive solutions of nonlinear elliptic equations, Comm. Partial Differential Equations 8 (1981), 883-901.

9. M. Fila, Boundedness of global solutions for the heat equation with nonlinear boundary conditions, Comment. Math. Univ. Carolinae 30 (1989), 479-484.

10. M. Fila and P. Quittner, The blowup rate for the heat equation with a nonlinear boundary condition, Math. Methods Appl. Sci. 14 (1991), 197-205.

11. A. Friedman and B. McLeod, Blowup of positive solutions of semilinear heat equations, Indiana Univ. Math. J. 34 (1985), 425-477.

12. A. Friedman, Partial differential equations of parabolic type, Prentice-Hall, Englewood Cliffs, NJ, 1964.

13. Y. Giga and R. V. Kohn, Asymptotic self-similar blowup of semilinear heat equations, Comm. Pure Appl. Math. 38 (1985), 297-319.

14. Characterizing blowup using similarity variables, Indiana Univ. Math. J. 36 (1987), 425-447.

15. _ Nondegeneracy of blowup for semilinear heat equations, Comm. Pure Appl. Math. 42 (1989), 845-884.

16. D. Gilbarg and N. S. Trudinger, Elliptic partial differential equations of second order, Springer-Verlag, New York, 1983.

17. O. A. Ladyzhenskaja, V. A. Solonnikov, and N. N. Ural'ceva, Linear and quasi-linear equations of parabolic type, Amer. Math. Soc. Transl. (2) 23 (1968).

18. H. A. Levine and L. E. Payne, Nonexistence theorems for the heat equation with nonlinear boundary conditions and for the porous medium equation backward in time, J. Differential Equations 16 (1974), 319-334.

19. H. A. Levine and R. A. Smith, A potential well theory for the heat equation with a nonlinear boundary condition, Math. Methods Appl. Sci. 9 (1987), 127-136.

20. W. Liu, The blowup rate of solutions of semilinear heat equations, J. Differential Equations 77 (1989), 104-122.

21. _ Blowup behavior for semilinear heat equations: multi-dimensional case, IMA preprint series no. 711, 1990.

22. P. Quittner, On global existence and stationary solutions for two classes of semilinear parabolic problems, Centre de recherche de mathematiques de la decision, Universite Paris IX, preprint 9208, 1992.

23. A. A. Samarskii, On new methods of studying the asymptotic properties of parabolic equations, Proc. Steklov Inst. Math. 158 (1983),165-176.

24. W. Walter, On existence and nonexistence in the large of solutions of parabolic differential equations with a nonlinear boundary condition, SIAM J. Math. Anal. 6 (1975), 85-90.

25. F. B. Weissler, Existence and non-existence of global solutions for a semilinear heat equation, Israel J. Math. 38 (1981), 29-40.

26. 38 (1985), 291-295.

27. H. M. Yin, Blowup versus global solvability for a class of nonlinear parabolic equations, Nonlinear Anal. TMA (to appear).

Department of Mathematics, University of Notre Dame, Notre Dame, Indiana 46556

E-mail address: Bei.Hu@nd.edu

E-mail address, Hong-Ming.Yin@nd.edu 